peptides of SV40 particles with those in uninfected monkey cells and monkey cells infected by this virus, in an apparently successful attempt to identify polypeptides which may well be specified by SV40.

Walter et al., like most other groups who have electrophoresed on gels the polypeptides liberated when SV40 particles are disrupted, find that two polypeptides with molecular weights of about 44,000 and 31,000 account for about 83 per cent of the total polypeptide of the virions. In addition they find as many as twelve other minor polypeptides in preparations of disrupted virions, at least some of which may well be coded by the host cell and others may be degradation products.

Of the protein made in cells after infection, about 10 per cent proves on electrophoresis to be identical to the two major polypeptides of the virus particles. Almost all of the other proteins made in infected cells can also be found in uninfected cells and must presumably be specified by the cell genome. But about 0.5 per cent of the protein which has accumulated at late times after infection can be resolved on electrophoresis into three polypeptides, the molecular weights of which can be estimated to be $70,000,60,000$ and 8,000 .

These three polypeptides are not found in extracts of uninfected cells, neither are they components of the SV40 particles and, therefore, adopting the cumbersome but familiar terminology devised by investigators of polio virus replication, Walter et al. designate them respectively as NVP1, NVP2 and NVP3. The first two of these three polypeptides are apparently accumulated in the nuclei of infected cells, and polypeptides of similar molecular weights can also be detected in Balb/3T3 cells infected with polyoma virus.

Walter et al. believe that the two major virion proteins, which can readily be detected in infected cells, are primary gene products, for the kinetics of their labelling and their half-lives argue against the idea that these two molecules are derived by cleavage maturation from a larger precursor molecule. Furthermore, because they comprise 10 per cent of the protein made in infected cells they are probably specified by the viral genome. The three nonvirion proteins-NVP1, NVP2 and NVP3-also might be coded by the virus because they are restricted to infected cells. If that is so, these polypeptides, together with the two virion proteins, account for the total coding capacity of the viral DNA.

As Walter et al. cryptically remark, the facts that studies with temperature sensitive mutants of polyoma virus indicate that they are two and possibly three early genes, and that SV40 infected cells acquire three new antigens ( $\mathrm{T}$ anti- gen, $U$ antigen and transplantation antigen), may not "represent a fortuitous agreement" with their detection of three non-virion polypeptides in infected cells. Of course, if these three polypeptides really are specified by the viral genome one or more of them must presumably be involved in maintaining the transformed cell phenotype of non-permissive cells transformed by SV40 and the identification of the "transforming" gene product is the ultimate goal of this work.

It would be wrong, however, to create the impression that the hunt is about to make a kill, because the alternative possibility, namely that one, two or all of the non-virion polypeptides are coded by cell genes, the expression of which is specifically induced by SV40 infection, has not in any rigorous way been excluded by the data obtained to date.

\section{INSULIN \\ Binding Process}

from a Correspondent

THE interaction of insulin with membranes is now attracting a great deal of interest, particularly because of recent work on the nature of the interaction of the hormones of insulin with liver plasma membranes and with membranes from isolated fat cells. This work is also taking place at a time when the three-dimensional structure of insulin has been elucidated so that there is increasing speculation about the nature of the residues on the surface of the insulin molecule which may be important in the binding process.

Set against this background the recent report by Gavin, Roth, Gen and Frey- chet (Proc. US Nat. Acad. Sci., 69, 747 ; 1972) concerning insulin receptors that have been identified on human lymphocytes is particularly interesting. These authors have shown that insulin and some of its derivatives can bind to human lymphocytes. The experiments have been carried out by measuring the displacement of insulin labelled with iodine-125, bound to lymphocyte membranes by insulins of various species and insulin derivatives (such as insulin lacking the $\mathrm{C}$-terminal alanine or the $8 \mathrm{C}$ terminal acids of the $\mathrm{B}$ chains). The manner in which these substances bind to lymphocytes very closely resembles the way in which they stimulate glucose oxidation in adipose tissue cells, and the results suggest important analogies between binding sites for insulin on human lymphocytes, on fat cells and on liver cells with regard to the kinetic properties and temperature dependence of the binding reaction.

Whether insulin has an important physiological role in lymphocyte metabolism is still largely open to question, but a recent report by Hadden and his colleagues (Nature New Biology, 235, $174 ; 1971)$ suggests that insulin may at least affect a lymphocyte plasma membrane ATPase. It may be that even if lymphocytes are not a very usual target tissue for insulin, typical receptor sites concerned with the binding of this hormone to cells are nevertheless present. Because lymphocytes are relatively easily obtained from human blood they may, as Gavin et al. suggest, be an ideal cell system in which to study insulin binding in man. Clearly work of this kind may have important implications for the further study of metabolic diseases such as diabetes, and it ought to be greatly extended.

\title{
Large Japanese Air Shower Examined
}

THE data on the very large extensive air shower which was reported last year by Suga et al. of the Institute for Nuclear Study, University of Tokyo (Phys. Rev. Lett., 27, 1604 ; 1971), are subjected to a critical analysis in next week's Nature Physical Science by Garmston and Watson of the University of Leeds. Suga and his colleagues claimed that the shower contained about $2 \times 10^{12}$ particles at sea level-where it was detected with an array of scintillator detectors and spark chambers. The Japanese group suggested, on this basis, that the energy of the primary cosmic ray responsible for initiating the shower at the top of the atmosphere was about $4 \times 10^{21} \mathrm{eV}$, the largest ever recorded.

Garmston and Watson take issue with this conclusion and propose that the data presented by the Japanesegroup last year are indicative of a primary energy of between $4 \times 10^{19} \mathrm{eV}$ and $1.4 \times 10^{20} \mathrm{eV}$, a factor of between 10 and 100 less than claimed. They say, for example, that depending on the shape assumed for the integral spectrum of the primary cosmic rays, only between $2 \times 10^{-3}$ and $4 \times 10^{-5}$ showers with an energy as high as $4 \times 10^{21} \mathrm{eV}$ would be expected during the running time of the Tokyo air shower array ( 3 years), taking into account the area covered by the detectors.

One of the difficulties in analysing the Tokyo air shower is that the core of the shower fell outside the area enclosed by the detectors, and it is therefore difficult to estimate the size of the shower and hence its energy. Garmston and Watson also examine very closely the energy spectrum which the Tokyo group assumes for the muons in the air shower and the way in which the density of air shower particles varies across the array itself. 